\title{
Flexor Tenorrhaphy Using Absorbable Suture Materials
}

\author{
Hyung Joo Kang, Dong Chul Lee, Jin Soo Kim, Sae Hwi Ki, Si Young Roh, Jae Won Yang \\ Department of Plastic and Reconstructive Surgery, Kwang-Myung Sung-Ae General Hospital, Gwangmyeong, Korea
}

Background Nonabsorbable sutures are favorable for repairing flexor tendons. However, absorbable sutures have performed favorably in an animal model.

Methods Two-strand sutures using the interlocking modified Kessler method with polydioxanone absorbable sutures 4-0 were used to repair completely ruptured flexor tendons in 55 fingers from 41 consecutive patients. The medical records of average 42 follow up weeks were analyzed retrospectively. The data analyzed using the chi-squared test, and Fisher's exact test was used for postoperative complications. The results were compared with those of other studies.

Results Among the index, middle, ring, and little fingers were injured in 9, 17, 16, and 13 fingers, respectively. The injury levels varied from zone 1 to 5 . Of the 55 digits in our study, there were $26(47 \%)$ isolated flexor digitorum profundus (FDP) injuries and 29 (53\%) combined FDP and with flexor digitorum superficialis injuries. Pulley repair was also conducted. Concomitant injuries of blood vessels and nerves were found in 17 patients (23 fingers); nerve injuries occurred in 5 patients (10 fingers). Two patients had ruptures (3.6\%), and one patient had two adhesions (3.6\%). Using the original Strickland criteria, all the patients were assessed to be excellent or good. Also, fibrosis and long-term foreign body tissue reactions such as stitch granuloma were less likely occurred in our study. Compared to the Cullen's report that used nonabsorbable sutures, there was no significant difference in the rupture or adhesion rates.

Conclusions Therefore, this study suggests that appropriate absorbable core sutures can be used safely for flexor tendon repairs.

Keywords Hand / Polydioxanone / Rupture / Tendon Injuries
Correspondence: Dong Chul Lee Department of Plastic and

Reconstructive Surgery, Kwang-Myung

Sung-Ae General Hospital, 36

Digital-ro, Gwangmyeong 423-711,

Korea

Tel: $+82-2-2680-7238$

Fax: +82-2-2615-7218

E-mail: ophand@gmail.com

Received: 8 Mar $2012 \bullet$ Revised: 23 May $2012 \bullet$ Accepted: 23 May 2012

pISSN: 2234-6163 • elSSN: 2234-6171 • http://dx.doi.org/10.5999/aps.2012.39.4.397 • Arch Plast Surg 2012;39:397-403

\section{INTRODUCTION}

Various sutures can be used to repair a ruptured flexor tendon. Since Avicenna first performed a tendon repair procedure, primary tendon repair has rarely been performed because primary tenorrhaphy is a controversial procedure [1]. Until the mid-20th century, using a tendon graft had been preferred over tendon repair for treating the "no man's land" section of the hand. Studies on flexor tendon healing of the digital sheath, however, have been performed since the beginning of the 20th century. Since Kleinert et al. [1] conducted primary repair for a flexor tendon rupture in the no man's land, tendon repair has been commonly performed on flexor tendon ruptures of the fingers; studies on the repair of the flexor tendon have been also conducted. Suture materials used for tendon repair have been developed over time. Many surgeons prefer sutures made of nonabsorbable materials such as nylon, braided polyester, and polypropylene [2]. However, Trail et al. [3] reported that using sutures made of nonab-

Copyright $(\odot 2012$ The Korean Society of Plastic and Reconstructive Surgeons

This is an Open Access article distributed under the terms of the Creative Commons Attribution Non-Commercial License (http://creativecommons.org/

licenses/by-nc/3.0/) which permits unrestricted non-commercial use, distribution, and reproduction in any medium, provided the original work is properly cited.

www.e-aps.org 
sorbable materials may cause stitch granuloma, fibrosis, triggering, and other complications because the body responds to the stimulation of the foreign suture material [4]. To overcome the aforementioned disadvantages of nonabsorbable suture materials, the use of absorbable suture materials have been considered. In 1995, O’Broin et al. [5] performed flexor tendon repair in an animal model using polydioxanone (PDS, Ethicon, San Angelo, TX, USA) absorbable sutures.

In this study, the authors repaired flexor tendons using absorbable sutures, and compared the clinical results with those of the published articles on flexor tendon repair using nonabsorbable sutures. This study was undertaken to determine the safety and efficacy of use of appropriate absorbable core sutures for finger flexor tendon repair in zones 1 to 5 .

\section{METHODS}

This study includes 41 cases, from June 2005 to December 2011, with a total of 55 fingers that had completely ruptured flexor tendons due to 15 cases (17 fingers) of crushing injury and 26 cases ( 38 fingers) of deep laceration of the hand. To minimize the influence of other variables, patients who had concomitant injuries to the bone or joint, completely amputated fingers, significant soft tissue defects, injuries to the flexor pollicis longus, and palmaris longus, or an avulsion injury of the flexor digitorum profundus (FDP) were excluded. Patients with blood vessel or nerve injuries were included. The concomitant injuries consisting of both blood vessels and nerves were found in 17 patients ( 23 fingers), and 5 patients ( 10 fingers) presented with only nerve injuries. Of the 41 patients, there were 32 men and 9 women, with an average age of 38 years (range, 16 to 67 years). Among the injured fingers, 9, 17, 16, and 13 injuries were identified in the index finger, middle finger, ring finger, and little finger, respectively. Middle finger injuries accounted for the majority of the injuries, and the injury levels varied from Verdan's flexor tendon zones 1 to zone 5 (Table 1). The majority of the patients $(73 \%)$ had injuries in zone 1 or zone 2 . Of the 55 injured digits, there were 26 (47\%) isolated FDP injuries, and 29 (53\%) FDP combined with flexor digitorum superficialis (FDS) injuries. The mean follow-up period was 42 weeks (range, 3 months to 5 years).

\section{Surgical methods and rehabilitation}

All surgeries were performed under a brachial plexus block. The Bruner zig-zag incision was used to expose the ruptured proximal tendon and the severed plane of the distal tendon. Debridement was minimally performed on the margin of the ruptured tendon. Subsequently, a core suture was inserted using the 2-strand interlocking modified Kessler method. The core sutures used were PDS 4-0 absorbable sutures. The circumferential repair procedure was a simple over-and-over running epitendinous suture technique using PDS 4-0 sutures. In the combined injuries, the FDS tendons were repaired with the same 2-strand interlocking modified Kessler technique. Pulley repairs were also performed in the 15 cases of pulley ruptures. In 12 cases, the A4 pulley ruptured with the flexor tendon at the injured site, so we

\begin{tabular}{|c|c|c|c|c|c|}
\hline $\mathrm{Pt}$ & Sex/Age & Injury & Vector & $\begin{array}{l}\text { Injury } \\
\text { levels }\end{array}$ & Results \\
\hline 1 & $\mathrm{M} / 54$ & Rt. LF & Glass & Zone 2 & Excellent \\
\hline 2 & $F / 33$ & Lt. RF & Knife & Zone 2 & Excellent \\
\hline 3 & M/33 & Rt. MF & Door & Zone 1 & Good \\
\hline 4 & $F / 34$ & Rt. RF & Knife & Zone 1 & Excellent \\
\hline 5 & $\mathrm{M} / 51$ & Rt. LF & Glass & Zone 2 & Excellent \\
\hline 6 & $\mathrm{~F} / 31$ & Rt. LF & Knife & Zone 1 & Excellent \\
\hline 7 & $\mathrm{M} / 30$ & Rt. IF, MF, RF, LF & Glass & Zone 5 & Good \\
\hline 8 & $\mathrm{M} / 16$ & Rt. MF & Metal & Zone 1 & Excellent \\
\hline 9 & $M / 24$ & Lt. IF & Metal & Zone 3 & Excellent \\
\hline 10 & $\mathrm{~F} / 67$ & Rt. IF & Glass & Zone 1 & Excellent \\
\hline 11 & $\mathrm{M} / 28$ & Lt. MF & Glass & Zone 2 & Good \\
\hline 12 & $\mathrm{M} / 42$ & Rt. LF & Door & Zone 2 & Good $^{\text {a) }}$ \\
\hline 13 & $F / 22$ & Rt. LF & Glass & Zone 2 & Excellent \\
\hline 14 & $\mathrm{M} / 37$ & Rt. MF, RF & Knife & Zone 2 & Good \\
\hline 15 & $M / 34$ & Lt. MF & Cylinder & Zone 1 & Good $^{b)}$ \\
\hline 16 & $\mathrm{M} / 48$ & Rt. IF, MF, RF & Glass & Zone 5 & Good \\
\hline 17 & $\mathrm{M} / 27$ & Lt. MF & Knife & Zone 1 & Excellent \\
\hline 18 & $\mathrm{M} / 22$ & Lt. IF, MF, RF, LF & Glass & Zone 5 & Good \\
\hline 19 & $\mathrm{M} / 27$ & Rt. RF & Glass & Zone 2 & Excellent \\
\hline 20 & $\mathrm{M} / 39$ & Rt. MF, RF, LF & Glass & Zone 3 & Good \\
\hline 21 & $\mathrm{M} / 32$ & Lt. IF & Metal & Zone 1 & Excellent \\
\hline 22 & $\mathrm{M} / 12$ & Lt. MF & Glass & Zone 5 & Good \\
\hline 23 & $\mathrm{M} / 49$ & Lt. RF & Glass & Zone 5 & Excellent \\
\hline 24 & $\mathrm{M} / 41$ & Lt. RF & Glass & Zone 3 & Excellent \\
\hline 25 & $\mathrm{M} / 34$ & Rt. RF & Metal & Zone 1 & Excellent \\
\hline 26 & $\mathrm{M} / 45$ & Lt. MF, RF & Glass & Zone 3 & Good $^{c)}$ \\
\hline 27 & F/32 & Rt. RF & Knife & Zone 1 & Excellent \\
\hline 28 & $\mathrm{M} / 48$ & Lt. MF & Knife & Zone 1 & Good \\
\hline 29 & $\mathrm{M} / 37$ & Rt. LF & Glinder & Zone 1 & Good \\
\hline 30 & $\mathrm{M} / 42$ & Rt. IF & Knife & Zone 1 & Good \\
\hline 31 & $\mathrm{M} / 34$ & Rt. LF & Knife & Zone 2 & Excellent \\
\hline 32 & $M / 43$ & Lt. MF & Metal & Zone 1 & Excellent \\
\hline 33 & $\mathrm{M} / 30$ & Lt. RF & Metal & Zone 3 & Excellent \\
\hline 34 & $F / 56$ & Rt. MF, RF & Metal & Zone 2 & Excellent \\
\hline 35 & $\mathrm{M} / 54$ & Rt. LF & Metal & Zone 2 & Excellent \\
\hline 36 & $M / 64$ & Rt. LF & Glass & Zone 2 & Excellent \\
\hline 37 & $\mathrm{~F} / 57$ & Rt. IF & Glass & Zone 2 & Excellent \\
\hline 38 & $\mathrm{M} / 44$ & Lt. LF & Metal & Zone 2 & Excellent \\
\hline 39 & $\mathrm{M} / 42$ & Lt. MF & Knife & Zone 2 & Excellent \\
\hline 40 & M/39 & Rt. IF, MF & Metal & Zone 3 & Excellent \\
\hline 41 & $F / 35$ & Lt. RF & Glass & Zone 2 & Excellent \\
\hline \multicolumn{6}{|c|}{$\begin{array}{l}\text { Pt, patient; Rt., right; Lt., left; IF, index finger; MF, middle finger; RF, ring finger; LF, } \\
\text { little finger; PL, palmaris longus. } \\
\text { a) Ruptured at } 34 \text { days after repair and immediately re-repaired (delayed tenorrhaphy). } \\
\text { b) Ruptured at } 88 \text { days after repair and PL tendon graft performed. }{ }^{c} \text { Tenolysis } \\
\text { performed } 6 \text { months after surgery. }\end{array}$} \\
\hline
\end{tabular}


repaired the pulley after repairing the flexor tendon. In 3 cases, the pulley systems were intact, but prevented the exposure of the ruptured flexor tendon ends, so we cut the pulleys and repaired the flexor tendon; we then also repaired the pulleys. In the concomitant blood vessel and nerve injury cases, both vascular anastomosis and nerve repair were performed under a microscope using 10-0 nylon sutures. After the skin repair, a dorsal protective splint was applied while the wrist was in the neutral position, the metacarpophalangeal joints were at $90^{\circ}$ flexion, and the proximal interphalangeal (PIP) and distal interphalangeal joints (DIP) were straight. On postoperative day 1 , dynamic active exercises for the fingers were performed with a dorsal splint protecting the wrist and without the assistance of rubber bands. The rehabilitation process consisted of active extension and active flexion according to early active motion protocols $[6,7]$. The patients were strictly advised not to passively stretch the repaired tendons, not to remove the splint unless instructed, and avoid holding the hand in a dependent position. The patients were encouraged to perform active flexion and extension by themselves as much as possible given their tolerance for pain. The splint was removed 4 weeks after the operation in most cases.

\section{Evaluation methods}

For comparing flexor tendon repair using nonabsorbable sutures with that of absorbable sutures, we referred to a study by Cullen et al. [6] for calculating the rupture rate as a percentage, assessing range of motion (ROM), complications, and rehabilitation methods. Similar to the Cullen's study [6], we analyzed the results of this study according to a retrospective review of the patients' charts, and the results were evaluated using the records of the evaluation of ROM at the final treatment.

In this study, the ROM was assessed using the Strickland system (excellent: $85 \%$ to $100 \%, 150^{\circ}$ to $175^{\circ}$; good: $70 \%$ to $84 \%$, $125^{\circ}$ to $149^{\circ}$; fair: $50 \%$ to $69 \%, 90^{\circ}$ to $124^{\circ}$; poor: $0 \%$ to $49 \%, 0^{\circ}$ to $\left.90^{\circ}\right)$. The Strickland system sums the degrees of active flexion at the DIP joint and the PIP joint and subtracts the degrees of the extension deficits. The results are compared with an ideal of $175^{\circ} \mathrm{ROM}[8]$ (Table 2). The repair rupture rate was then calculated.

\section{Table 2. Strickland and Glogovac criteria [8] of evaluation}

\begin{tabular}{|lcc|}
\hline Grade & $\begin{array}{c}\text { Total active range of } \\
\text { motion }^{\text {a) }}\left({ }^{\circ}\right)\end{array}$ & $\begin{array}{c}\text { Functional } \\
\text { return }(\%)\end{array}$ \\
\hline Excellent & $>150$ & $85-100$ \\
Good & $125-149$ & $70-84$ \\
Fair & $90-124$ & $50-60$ \\
Poor & $<90$ & $0-49$ \\
\hline a) Sum of the active range of motion of the distal interphalangeal joints and proximal \\
interphalangeal joints.
\end{tabular}

Statistical analysis using SPSS ver 19.0 (IBM Co., Armonk, New York, USA) was performed for the postoperative complications, and the results were compared with those of other studies. The statistical tests used were chi-squared testing and Fisher's exact test. Statistical significance was assumed for $\mathrm{P}<0.05$.

\section{RESULTS}

Among the 55 injured fingers of the 41 patients, repair rupture of the tendon was shown in 2 fingers of 2 patients; subsequently one patient underwent secondary surgery, specifically delayed tenorrhaphy, and the other underwent a palmaris longus tendon graft. The repair rupture rates were two out of 55 (3.6\%) for the absorbable core suture group and two out of 38 (5.3\%) for the nonabsorbable group of the Cullen's report [6]. Using Fisher's exact test, this did not reach statistical significance $(\mathrm{P}=0.580)$. All the ruptures were of the FDP tendon only, with one rupture in zone 2 and one in zone 1 . This is out of the total of 55 fingers in our study, in which there were $26(47 \%)$ isolated FDP injuries and 29 (53\%) combined FDP and FDS injuries. In one case, repair rupture was identified 34 days after the initial repair and required delayed tenorrhaphy. In the other case, repair rupture occurred 88 days after the initial repair, and required a palmaris longus (PL) tendon graft. Repair rupture was caused by hyperextension and inflammation after surgery.

The functional outcome was assessed using the original Strickland criteria. In the absorbable core suture group ( 55 digits, 84 tendons) in our study, the results were $53 \%$ excellent, $47 \%$ good, $0 \%$ fair, and $0 \%$ poor. In the nonabsorbable core suture group (38 digits, 70 tendons) of the Cullen's report [6], the results were $71 \%$ excellent, $6.5 \%$ good, $3.5 \%$ fair, and $19 \%$ poor. The chi-squared tests confirmed that these small differences were not statistically significant $(\mathrm{P}=0.001)$.

In the absorbable core suture group in our study, 2 (3.6\%) of the 55 injured fingers required tenolysis, whereas 2 (5.3\%) of the 38 in the nonabsorbable core group had required tenolysis in Cullen's report [6]; the difference did not reach statistical significance $(\mathrm{P}=0.580)$.

A long-term foreign body reaction such as stitch-induced granuloma was not found in either the absorbable suture group in our study or the nonabsorbable suture group in Cullen's report [6]. According to an article by Pabari et al. [4], the evidence of stitch-induced granuloma is painless cystic swelling in the palm under a previous scar with no history of further trauma. In our group, there were no symptoms or signs of stitch-induced granuloma in any of our cases.

According to studies on flexor tendon repair using nonabsorbable sutures, the repair rupture rate has been shown to vary from 
2 to $10 \%$ [9-12]. Cullen et al. [6] reported a rupture rate of $5.3 \%$, and Caulfield et al. [10] reported a rupture rate of $2 \%$ using nonabsorbable sutures. The results of the present study showed that the rupture rate was similar, and the ROM was also satisfactory, in flexor tendon repairs using absorbable sutures compared to the conventional methods using nonabsorbable suture materials (Table 3 ).

\section{Case 1}

A 33-year-old woman visited our hospital due to soft tissue damage on the PIPjoint of the left ring finger to the volar and ulnar region, and a complete rupture of the FDP tendon caused by a knife-induced injury. No injury of the artery, vein, or nerve was found under microscopic examination. The patient underwent a surgical repair of the FDP tendon. The patient began active exercises from the first postoperative day. Six months after reconstructing the flexor tendon, the ROM was assessed as excellent at $170^{\circ}$ according to the Strickland system. No repair rupture occurred (Figs. 1, 2).

\section{Case 2}

A 43-year-old man visited our hospital presenting with soft tissue damage on the PIP joint to the fingertip of the left middle finger to the volar ulnar side, a complete rupture of the FDP tendon caused by a metal-induced injury. Blood circulation was intact. No injury of the artery, vein, or nerve was found under

\begin{tabular}{|c|c|c|}
\hline Outcomes & Absorbable (PDS) & Nonabsorbable \\
\hline Rupture rate (\%) & 3.6 & $2-10$ \\
\hline Range of motion (Strickland's) (\%) & 100 & $53-85$ \\
\hline
\end{tabular}

microscopic examination. The patient underwent surgical repair of the FDP tendon. The patient began active exercises from the first postoperative day. Three months after reconstructing the flexor tendon, the ROM was assessed as excellent at $165^{\circ}$ according to the Strickland system. No repair rupture occurred (Figs. 3, 4).

\section{DISCUSSION}

When choosing a suture material for flexor tendon repair, the suture's tensile strength, knot-holding ability, stretch, biocompatibility, manipulation, and complication rate should be considered. Compared to nonabsorbable sutures, the use of absorbable sutures may avoid problems associated with longterm foreign body implantation such as excessive fibrosis and stitch granuloma, but maintaining adequate tensile strength during the early reconstruction period remains a challenge due

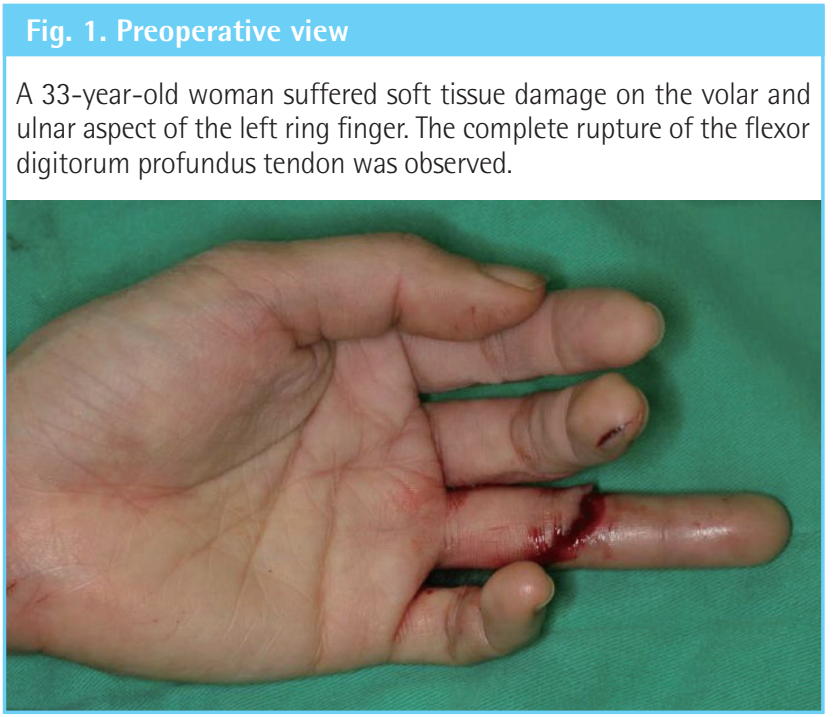

\section{Fig. 2. Postoperative views}

(A) Assessment of the postoperative tendon function six months after the surgery. The functional outcome was excellent according to the Strickland system, although mild flexion limitation on the distal interphalangeal joint was observed. (B) Full extension without postoperative extension lag.
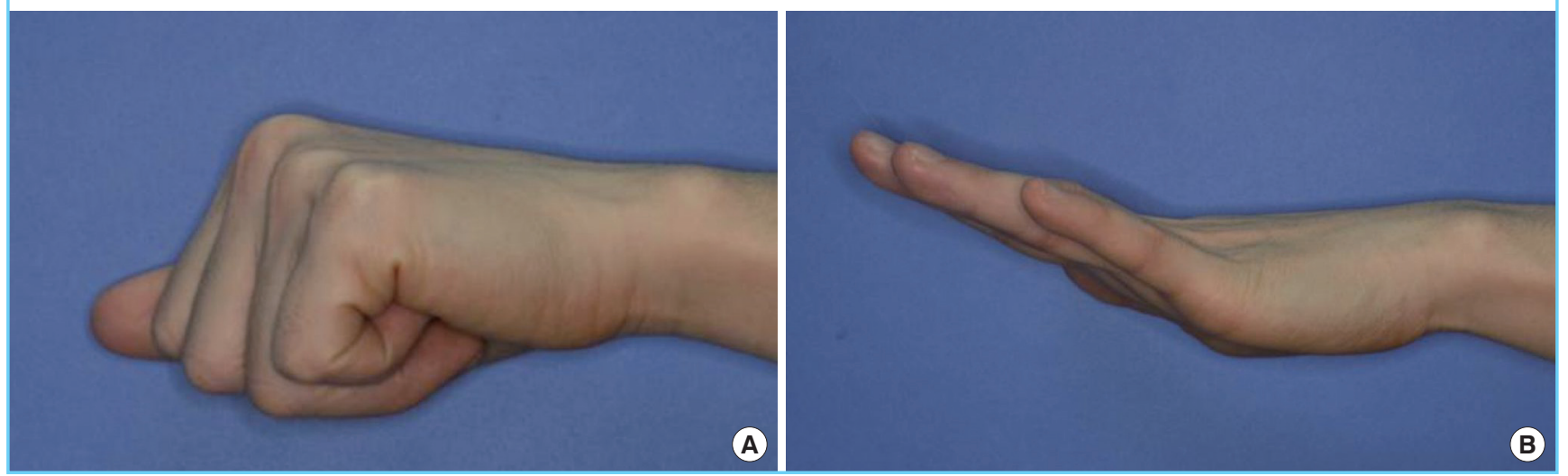
to their absorption into the body $[3,5]$. Thus, using absorbable sutures has been controversial. Meanwhile, nonabsorbable sutures are widely used due to the advantages of easy handling, good biocompatibility, and minimal loss of tensile strength after knotting. However, it has been reported that nonabsorbable sutures can cause complications such as granuloma, fibrosis, and triggering due to the stimulation of a suture [4]. Despite the aforementioned complications, nonabsorbable suture materials have been used for tendon repair, depending on the preferences of surgeons. In 1940, Siler [13] repaired a flexor tendon using silk, which is a nonabsorbable suture material. In 1970, Miller [14] repaired flexor tendons using braided polyester sutures. In addition, Strickland [15] have also repaired flexor tendons using nonabsorbable suture materials $[3,16]$. Since then, flexor tendon repair using absorbable suture materials has been introduced, and the recent studies on using absorbable sutures for tendon repair have been encouraging given the disadvantages

\section{Fig. 3. Preoperative view}

A 43-year-old man suffered soft tissue damage on the proximal interphalangeal joint to the fingertip of the left middle finger to the volar and ulnar region. The complete rupture of the flexor digitorum profundus tendon was observed.

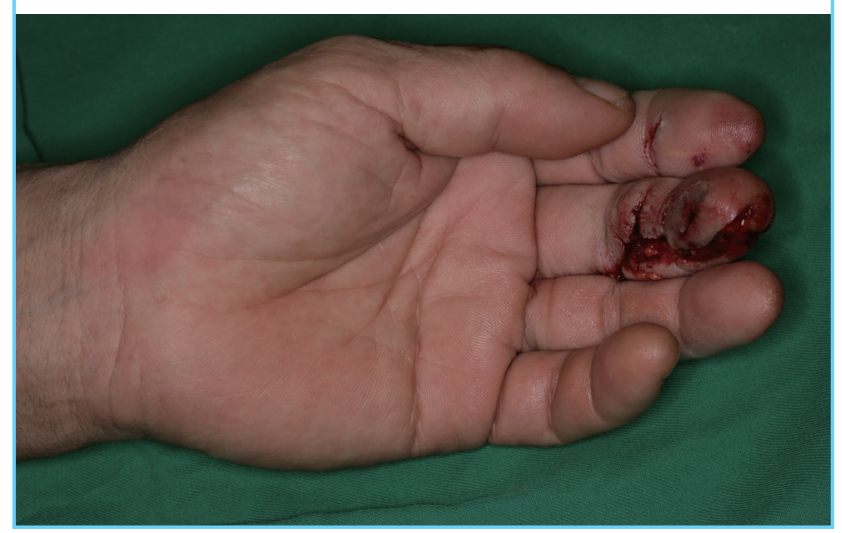

of nonabsorbable suture materials. Synthetic absorbable suture materials such as polyglycolic acid or polyglactin polymer were used when absorbable sutures were first introduced. However, they were inconvenient due to their rigidity and inflexibility [17]. Monofilament fiber PDS has been recently used for flexor tendon repairs. The results of an animal experiment reported by O'Broin [5] showed that PDS was not only flexible and strong, but also had high breaking strength and minimal foreign body reaction. PDS retained its breaking strength for 9 weeks, which is a relatively long time. Thus, it was able to compensate for the disadvantages of absorbable sutures such as unpredictable maintenance of tensile strength $[5,18,19]$. Furthermore, PDS has the advantages of excellent wound support ability, less inflammatory responses caused by the decreased delivery of pathogenic bacteria, as well as minimal toxicity and easier handling. Thus, PDS has been widely used for flexor tendon repairs [17]. Chusak and Dibbell [17] reported that the use of PDS sutures during surgery had good outcomes in all the following assessment items: pliability, strength, ease of passage, ease of tying, knot security, overall handling convenience, and fraying; in addition, PDS achieved complete healing in all the cases with a follow-up of 42 days or more.

Surgeons have been reluctant to use sutures made of absorbable materials because of the significant loss in suture strength [2]. However, O'Broin et al. [5] reported that compared to the tensile strength of nonabsorbable sutures (Prolene, Ethicon), PDS sutures had higher tensile strength at the beginning of an operation and had similar tensile strength at the end of the second week; at this stage, there was no significant difference in suture strength between tendons repaired with PDS and Prolene. At 4 weeks, PDS has lost 50\% of its original strength, and full tensile strength was lost at week 9. The tensile strength of absorbable sutures gradually decreases with time. However, the

\section{Fig. 4. Postoperative views}

(A) Assessment of postoperative tendon function three months after the surgery. (B) Excellent total active range of motion. Full extension without postoperative extension lag.
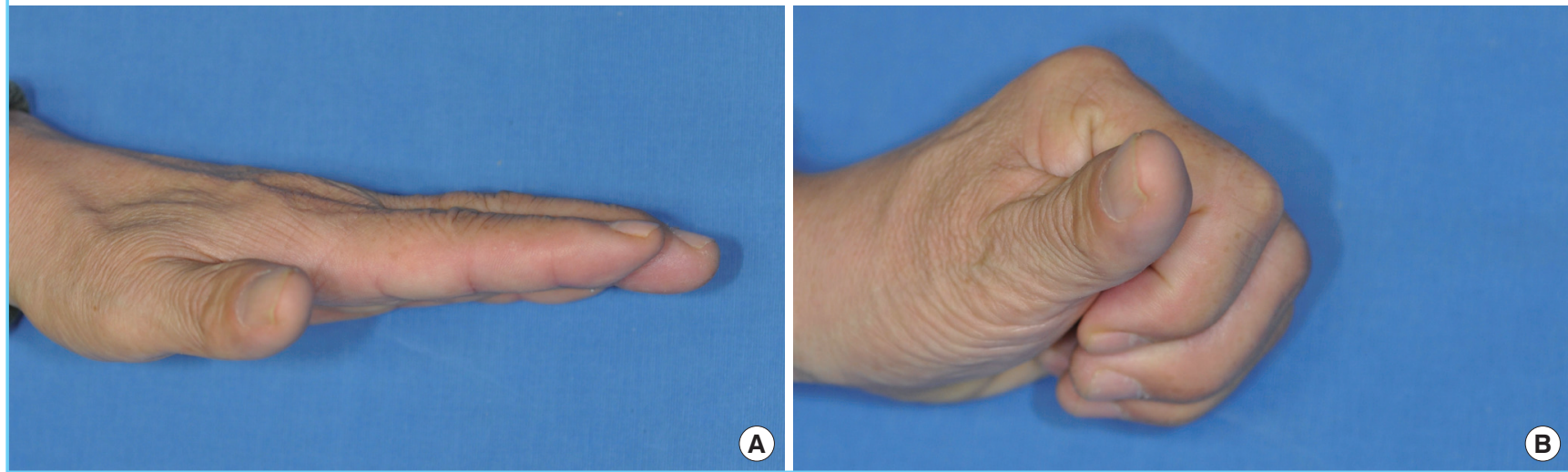
tensile strength of the tendon itself decreases until three days after the tendon repair, and thereafter the tendon tensile strength gradually increases; at 4 weeks, the repair strength of the tendon is approximately 8 times stronger than the repair strength at the time the sutures were applied. The fact that PDS had lost half its tensile strength is of no significance in terms of the total strength of a 4-week old repair [5]. Due to this advantage, PDS has been used for flexor tendon repair at our hospital.

Complications such as repair rupture and adhesion, and joint stiffness may occur after flexor tendon repair [9,20]. Re-rupture of a repaired tendon is due to unplanned high loads that exceed the tolerance of the repaired tendon. It may also occur from early excessive exercise or unexpected finger motion such as sudden gripping or falling down on outstretched hands without wearing a protective splint [9]. Among the patients who underwent flexor tendon repair using PDS, repair rupture occurred in 2 cases, which were immediately repaired. In one case, the repair rupture was identified 34 days after the initial repair and required delayed tenorrhaphy. In the other case, the repair rupture occurred 88 days after the initial repair and required a PL tendon graft. Excessive gripping, hyperextension, and inflammation caused the repair ruptures after surgery When the surface of the flexor tendon was observed during the secondary surgery, no adhesion was found in the patient who underwent delayed tenorrhaphy 34 days after the initial repair. However, severe adhesion of the FDP tendon with the nearby soft tissues and partial loss of the tendon caused by infection were found in the patient who had a ruptured tendon 88 days after the initial tenorrhaphy. This rupture is likely to be attributed to the decreased tensile strength caused by an infection, which finally resulted in tendon necrosis.

The patients of the Cullen's study [6] underwent flexor tendon repairs using nonabsorbable sutures such as Ticron Ticron (Covidien plc, Dublin, Ireland) and Prolene. Cullen et al. [6] reported that repair rupture occurred 10 days after surgery in a patient who performed excessive hand gripping within a few days after the operation, and 14 days after surgery in another patient who excessively exercised beyond the recommended exercise criteria. In this study using PDS, an absorbable suture material, rupture occurred in the subacute phase rather than in the early phase after surgery. On the other hand, in Cullen's report [6] using nonabsorbable suture materials, rupture occurred in the early phase after surgery. However, no significant difference in the rupture rate was found. Thus, the aforementioned result showed that the conventional belief that the rupture rate increases if absorbable suture materials are used appears to be wrong. In addition, in our study, tenolysis was required in only one patient due to tendon adhesion, and the follow-up results showed recovery of normal ROM. In Cullen's report [6], 2 patients required tenolysis and showed a fair recovery of ROM 4 months after the secondary operation. In this study, no increase in the complication rate was found in the flexor tendon repair using absorbable suture materials, and functional outcomes were also satisfactory. Therefore, the use of absorbable suture materials is clinically meaningful.

This study has some limitations. The tendon repair methods and exercise regimens are not exactly the same in our study and Cullen's report [6]. The grade of the surgeons may not be similar between Cullen and the authors; but the skill of the surgeon was not a factor in this study because all of the repairs were performed by the senior authors. Regarding the repair method, the authors repaired the flexor tendons using the interlocking modified Kessler method, and as noted, Cullen et al. [6] repaired flexor tendons using the modified Kessler method. According to Mashadi and Amis [21], locking sutures do not improve either ultimate tensile strength or gap resistance; therefore, the interlocking modified Kessler method in our study had no major effect on the results, contrary to what we had expected. The authors were able to compare the study with Cullen's report [6] because both used similar repair methods and similar exercise regimens.

The authors repaired the flexor tendons using PDS on 55 fingers of 41 patients who presented with a completely ruptured flexor tendon from June 2005 to December 2011. The results were as follows: no complications such as stitch granuloma or triggering, which are problems of the existing nonabsorbable suture materials, occurred. The rate of complications such as repair rupture or adhesion was similar for PDS and nonabsorbable suture materials. Furthermore, less restriction of ROM and satisfactory functional recovery were obtained. Therefore, flexor tendon repair using absorbable suture materials can be useful in clinical practice.

\section{REFERENCES}

1. Kleinert HE, Spokevicius S, Papas NH. History of flexor tendon repair. J Hand Surg Am 1995;20:S46-52.

2. David GW. Flexor tendon injuries and reconstruction. In: Mathes SJ, Hentz VR, editors. Mathes plastic surgery. 2nd ed. Philadelphia: Saunders Elsevier; 2006. p.359-82.

3. Trail IA, Powell ES, Noble J. An evaluation of suture materials used in tendon surgery. J Hand Surg Br 1989;14:422-7.

4. Pabari A, Iyer S, Branford OA, et al. Palmar granuloma following flexor tendon repair using Ticron: a case for absorbable suture material? J Plast Reconstr Aesthet Surg 2011;64: 409-11. 
5. O’Broin ES, Earley MJ, Smyth H, et al. Absorbable sutures in tendon repair: a comparison of PDS with prolene in rabbit tendon repair. J Hand Surg Br 1995;20:505-8.

6. Cullen KW, Tolhurst P, Lang D, et al. Flexor tendon repair in zone 2 followed by controlled active mobilisation. J Hand Surg Br 1989; 14:392-5.

7. Small JO, Brennen MD, Colville J. Early active mobilisation following flexor tendon repair in zone 2. J Hand Surg Br 1989; 14:383-91.

8. Strickland JW, Glogovac SV. Digital function following flexor tendon repair in Zone II: a comparison of immobilization and controlled passive motion techniques. J Hand Surg Am 1980;5:537-43.

9. Tang JB. Clinical outcomes associated with flexor tendon repair. Hand Clin 2005;21:199-210.

10. Caulfield RH, Maleki-Tabrizi A, Patel H, et al. Comparison of zones 1 to 4 flexor tendon repairs using absorbable and unabsorbable four-strand core sutures. J Hand Surg Eur Vol 2008;33:412-7.

11. Elliot D, Moiemen NS, Flemming AF, et al. The rupture rate of acute flexor tendon repairs mobilized by the controlled active motion regimen. J Hand Surg Br 1994;19:607-12.

12. Moiemen NS, Elliot D. Primary flexor tendon repair in zone 1.J Hand Surg Br 2000;25:78-84.
13. Siler VE. Primary tenorrhaphy of the flexor tendons in the hand.J Bone Joint Surg Am 1950;32:218-25.

14. Miller JM. A new era of non-absorbable sutures. Exp Med Surg 1970;28:274-80.

15. Strickland JW. Development of flexor tendon surgery: twenty-five years of progress. J Hand Surg Am 2000;25:214-35.

16. Ikuta Y, Tsuge K. Postoperative results of looped nylon suture used in injuries of the digital flexor tendons. J Hand Surg Br 1985;10:67-72.

17. Chusak RB, Dibbell DG. Clinical experience with polydioxanone monofilament absorbable sutures in plastic surgery. Plast Reconstr Surg 1983;72:217-21.

18. Bourne RB, Bitar H, Andreae PR, et al. In-vivo comparison of four absorbable sutures: Vicryl, Dexon Plus, Maxon and PDS. Can J Surg 1988;31:43-5.

19. Wada A, Kubota H, Akiyama T, et al. Effect of absorbable polydioxanone flexor tendon repair and restricted active mobilization in a canine model. J Hand Surg Am 2001;26: 398-406.

20. Momeni A, Grauel E, Chang J. Complications after flexor tendon injuries. Hand Clin 2010;26:179-89.

21. Mashadi ZB, Amis AA. The effect of locking loops on the strength of tendon repair. J Hand Surg Br 1991;16:35-9. 